\title{
Pricing Extendible Options Using the Fast Fourier Transform
}

\author{
Siti Nur Iqmal Ibrahim, ${ }^{1,2}$ John G. O'Hara, ${ }^{3,4}$ and Nick Constantinou ${ }^{5}$ \\ ${ }^{1}$ Department of Mathematics, Faculty of Science, Universiti Putra Malaysia (UPM), 43400 Serdang, Selangor, Malaysia \\ ${ }^{2}$ Institute for Mathematical Research, Universiti Putra Malaysia (UPM), 43400 Serdang, Selangor, Malaysia \\ ${ }^{3}$ Centre for Computational Finance \& Economic Agents, University of Essex, Colchester CO4 3SQ, UK \\ ${ }^{4}$ Mathematical Sciences, University of KwaZulu-Natal, Westville Campus, Durban 3001, South Africa \\ ${ }^{5}$ Essex Business School, University of Essex, Colchester CO4 3SQ, UK \\ Correspondence should be addressed to Siti Nur Iqmal Ibrahim; iqmal@upm.edu.my
}

Received 20 February 2014; Revised 14 May 2014; Accepted 28 May 2014; Published 16 June 2014

Academic Editor: Wei-guo Zhang

Copyright (C) 2014 Siti Nur Iqmal Ibrahim et al. This is an open access article distributed under the Creative Commons Attribution License, which permits unrestricted use, distribution, and reproduction in any medium, provided the original work is properly cited.

\begin{abstract}
This paper applies the fast Fourier transform (FFT) approach, within the Black-Scholes framework, to the valuation of options whose time to maturity can be extended to a future date (extendible options). We determine the valuation of the extendible options as sums of expectations of indicator functions, leading to a semianalytic expression for the value of the options over a range of strikes. Compared to Monte Carlo simulation, numerical examples demonstrate that the FFT is both computationally more efficient and higher in accuracy.
\end{abstract}

\section{Introduction}

An extendible option allows its time to expiration to be extended to a future date for a fee with a different strike price at the extended expiration time. Longstaff [1] discusses a number of applications of extendible options, such as extendible bonds and warrants, and applications in real estate, demonstrating that extendible options are important in financial markets. For example, Dias and Rocha [2] price petroleum concessions using a mean-reverting framework including jumps for an extendible option. Extendible options can be classified as a holder- or writer-extendible call option or a holder- or writer-extendible put option. Options that are extended by their writer have no fee and they are more straightforward to price. To the best of our knowledge, Longstaff [1] was the first to study extendible options. Chung and Johnson [3] extend the work of Longstaff [1] to a general case where the holder or the writer can extend the option more than once and provide a general closed-form pricing solution for $n$-time extendible options.

In order to price options, many numerical techniques are available, such as Monte Carlo simulation and the Fourier transform method. The fast Fourier transform (FFT) was first introduced by Cooley and Tukey [4] to obtain the discrete Fourier transform (DFT) and its inverse. Implementing the DFT involves $N$ points where, for added efficiency, $N$ is a power of 2 and it is essential to have an extensively large $N$ data set, which reduces the number of computations from $O\left(N^{2}\right)$ to $O(N \ln N)$. Monte Carlo simulation often requires substantially more time to compute option prices, while the FFT, which was first implemented to option pricing by Carr and Madan [5], is often more straightforward and effective. This has been demonstrated by a number of authors, such as Pillay and O'Hara [6] and Ibrahim et al. [7]. Hence, the FFT has gained popularity as an effective option pricing methodology (see $[8,9]$ ), and a significant amount of research has extended this method to price various types of options, for instance Asian options [10], spread options [11], and power options [12]. The FFT technique is widely applicable and has been shown to perform well in terms of its speed and accuracy in some other affine models, such as stochastic volatility, stochastic interest rates, and jumps (see [13-15]).

In this paper, we review the pricing framework for extendible call options under the Black and Scholes [16] model first developed by Longstaff [1]. By following the work of Carr and Madan [5], we apply the FFT method to 
numerically compute the prices of extendible call options. For an effective implementation of the FFT, we provide an analytical representation of the characteristic functions for the extendible options. The usefulness of the FFT is that this technique gives a range of option prices for a collection of strike prices for a given maturity. In addition, we estimate the price of extendible options via Monte Carlo simulation for comparison.

The paper is organized as follows. Section 2 reviews the closed-form formulas for the holder-extendible call options under the Black-Scholes model as provided in Longstaff [1]. Section 2.1 develops a pricing procedure for holderextendible call options using the FFT approach where the characteristic functions are given, while Section 2.2 applies the Monte Carlo simulation using an Euler scheme and a Milstein scheme. Section 3 presents the numerical results, and Section 4 concludes the paper.

\section{Extendible Option}

In this section, we derive a closed-form solution for holderextendible call options as given by Longstaff [1], which is the focus of this paper. An analogous approach can be used to value extendible put options.

Let $(\Omega, \mathscr{F}, \mathbf{Q})$ be a probability space on which a Brownian motion, $W_{t}, 0 \leq t \leq T$, is defined, where $\mathscr{F}_{t}, 0 \leq t \leq T$, is the natural filtration generated by the Brownian motion. Suppose that $\mathbf{Q}$ is a risk-neutral measure under which the asset price process $S_{t}, 0 \leq t \leq T$, is governed by the following dynamics:

$$
d S_{t}=r S_{t} d t+\sigma S_{t} d W_{t}
$$

Within the Black-Scholes framework, the volatility $\sigma$ and the risk-free interest rate $r$ are assumed to be constant. Using Itô's lemma, the process followed by the logarithmic asset price $x_{t}:=\ln S_{t}$ is given by

$$
d x_{t}=\left(r-\frac{1}{2} \sigma^{2}\right) d t+\sigma d W_{t}
$$

This demonstrates that $x_{t}$ follows a generalized Wiener process with a constant drift $\left(r-(1 / 2) \sigma^{2}\right)$ and a constant volatility $\sigma$. Therefore, the change in $x$ between time $t$ and $T$ is normally distributed with a mean of $\left(r-(1 / 2) \sigma^{2}\right)(T-t)$ and a variance of $\sigma^{2}(T-t)$ or

$$
\ln S_{T} \sim N\left[\ln S_{t}+\left(r-\frac{1}{2} \sigma^{2}\right)(T-t), \sigma^{2}(T-t)\right] .
$$

Therefore, $S_{T}$ has a log-normal distribution. The valuation formula for a vanilla call option with a given underlying asset price $S_{t}$, strike price $K$, expiration time $T$, risk-free interest rate $r$, and volatility $\sigma$ is given as follows:

$$
C\left(S_{t}, K, T-t\right)=S_{t} N\left(d_{1}\right)-K e^{-r(T-t)} N\left(d_{2}\right),
$$

where

$$
\begin{aligned}
& d_{1}=\frac{\ln \left(S_{t} / K\right)+\left(r+(1 / 2) \sigma^{2}\right)(T-t)}{\sigma \sqrt{T-t}}, \\
& d_{2}=d_{1}-\sigma \sqrt{T-t}
\end{aligned}
$$

Let EC be the value of an extendible call option with time to expiration of $T_{1}$. At the time to expiration $T_{1}$, the holder of the extendible call can

(1) let the call expire worthless if $S_{T_{1}}<L$, or

(2) exercise the call and get $S_{T_{1}}-K_{1}$ if $S_{T_{1}}>H$, or

(3) make a payment of an additional premium $A$ to extend the call to $T_{2}$ with a new strike of $K_{2}$ if $L \leq$ $S_{T_{1}} \leq H$,

where $S_{T_{1}}$ is the underlying asset price and strike price at time $T_{1}, K_{1}$ is the strike price at time $T_{1}$, and Longstaff [1] refers to $L$ and $H$ as critical values, where $L<H$.

If at expiration time $T_{1}$ the option is worth more than the extendible value with a new strike price of $K_{2}$ for a fee of $A$ for extending the expiration time $T_{1}$ to $T_{2}$, then it is best to exercise; that is, $S_{T_{1}}-K_{1} \geq C\left(S_{T_{1}}, K_{2}, T_{2}-T_{1}\right)-A$. Otherwise, it is best to extend the expiration time of the option to $T_{2}$ and exercise when it is worth more than zero; that is, $C\left(S_{T_{1}}, K_{2}, T_{2}-T_{1}\right)-A>0$. Moreover, the holder of the option should be impartial between extending and not exercising at value $L$ and impartial between exercising and extending at value $H$. Therefore, the critical values $L$ and $H$ are unique solutions of $H-K_{1}=C\left(H, K_{2}, T_{2}-T_{1}\right)-A$ and $C\left(L, K_{2}, T_{2}-T_{1}\right)-A=0$. See Longstaff [1] and Gukhal [17] for an analysis of the conditions.

The price of the extendible call option is the discounted expectation of the payoff at time $T_{1}$ and is given by the following:

$$
\begin{aligned}
& \operatorname{EC}\left(S_{t}, K_{1}, T_{1}, K_{2}, T_{2}, A\right) \\
& =e^{-r\left(T_{1}-t\right)} \\
& \quad \times \mathbf{E}^{\mathbf{Q}}\left\{\max \left[S_{T_{1}}-K_{1}, C\left(S_{T_{1}}, K_{2}, T_{2}-T_{1}\right)-A, 0\right]\right\} \\
& =e^{-r\left(T_{1}-t\right)} \mathbf{E}^{\mathbf{Q}}\left[\left(S_{T_{1}}-K_{1}\right) \mathbf{1}_{\left\{S_{T_{1}}>H\right\}} \mid \mathscr{F}_{t}\right] \\
& +e^{-r\left(T_{1}-t\right)} \mathbf{E}^{\mathbf{Q}}\left[\left(C\left(S_{T_{1}}, K_{2}, T_{2}-T_{1}\right)-A\right)\right. \\
& \left.\quad \times \mathbf{1}_{\left\{L \leq S_{T_{1}} \leq H\right\}} \mid \mathscr{F}_{t}\right] .
\end{aligned}
$$

An example illustration of its payoff is provided in Figure 1.

Formulating the value of a call option, $C$ at time $T_{1}$ with a time to expiration extended to $T_{2}$, as the discounted conditional expected payoff,

$$
e^{-r\left(T_{2}-T_{1}\right)} \mathbf{E}^{\mathbf{Q}}\left[\max \left(S_{T_{2}}-K_{2}, 0\right) \mid \mathscr{F}_{t}\right]
$$

yields

$$
\begin{aligned}
\mathrm{EC} & \left(S_{t}, K_{1}, T_{1}, K_{2}, T_{2}, A\right) \\
= & e^{-r\left(T_{1}-t\right)} \mathbf{E}^{\mathbf{Q}}\left[\left(S_{T_{1}}-K_{1}\right) \mathbf{1}_{\left\{S_{T_{1}}>H\right\}} \mid \mathscr{F}_{t}\right] \\
& +e^{-r\left(T_{2}-t\right)} \mathbf{E}^{\mathbf{Q}}
\end{aligned}
$$




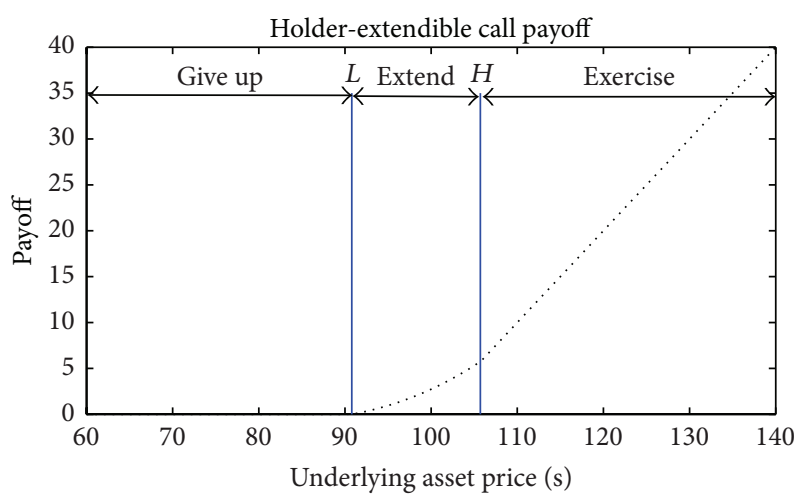

FIGURE 1: An example of the payoff function for an extendible call option for $K_{1}=100, K_{2}=105, r=0.08, \sigma=0.25, T_{1}=0.5, T_{2}=$ $0.75, A=1$.

$$
\begin{aligned}
& \times\left[\left(S_{T_{2}}-K_{2}\right) \mathbf{1}_{\left\{S_{T_{2}}>K_{2}\right\}}\left(\mathbf{1}_{\left\{S_{T_{1}} \geq L\right\}}-\mathbf{1}_{\left\{S_{T_{1}} \geq H\right\}}\right) \mid \mathscr{F}_{t}\right] \\
& -e^{-r\left(T_{1}-t\right)} \mathbf{E}^{\mathbf{Q}}\left[A\left(\mathbf{1}_{\left\{S_{T_{1}} \geq L\right\}}-\mathbf{1}_{\left\{S_{T_{1}} \geq H\right\}}\right) \mid \mathscr{F}_{t}\right]
\end{aligned}
$$

or

$$
\begin{aligned}
& \operatorname{EC}\left(S_{t}, K_{1}, T_{1}, K_{2}, T_{2}, A\right) \\
& =S_{t} \mathbf{E}^{\mathbf{Q}_{S}}\left[\mathbf{1}_{\left\{x_{T_{1}}>h\right\}}\right]-K_{1} e^{-r\left(T_{1}-t\right)} \mathbf{E}^{\mathbf{Q}}\left[\mathbf{1}_{\left\{x_{T_{1}}>h\right\}}\right] \\
& +S_{t} \mathbf{E}^{\mathbf{Q}_{S}}\left[\mathbf{1}_{\left\{x_{T_{1}} \geq l, x_{T_{2}} \geq k_{2}\right\}}\right]-K_{2} e^{-r\left(T_{2}-t\right)} \mathbf{E}^{\mathbf{Q}}\left[\mathbf{1}_{\left\{x_{T_{1}} \geq l, x_{T_{2}} \geq k_{2}\right\}}\right] \\
& -S_{t} \mathbf{E}^{\mathbf{Q}_{S}}\left[\mathbf{1}_{\left\{x_{T_{1}} \geq h, x_{T_{2}} \geq k_{2}\right\}}\right]+K_{2} e^{-r\left(T_{2}-t\right)} \mathbf{E}^{\mathbf{Q}}\left[\mathbf{1}_{\left\{x_{T_{1}} \geq h, x_{T_{2}} \geq k_{2}\right\}}\right] \\
& -A e^{-r\left(T_{1}-t\right)}\left\{\mathbf{E}^{\mathbf{Q}}\left[\mathbf{1}_{\left\{x_{T_{1}} \geq l\right\}}\right]-\mathbf{E}^{\mathbf{Q}}\left[\mathbf{1}_{\left\{x_{T_{1}} \geq h\right\}}\right]\right\},
\end{aligned}
$$

where $\mathbf{Q}_{S}$ is the spot price measure, $x_{t}=\ln S_{t}, k_{1}=\ln K_{1}, k_{2}$ $=\ln K_{2}, l=\ln L$, and $h=\ln H$.

Corollary 1. The price of an extendible call option with time to expiration $T_{1}$ and strike price $K_{1}$, whose expiration time may be extended to $T_{2}$ upon a payment of an additional premium $A$, with a new strike price $K_{2}$ is specified as

$$
\begin{aligned}
E C( & \left.S_{t}, K_{1}, T_{1}, K_{2}, T_{2}, A\right) \\
= & {\left[S_{t} N\left(a_{1}\right)-K_{1} e^{-r\left(T_{1}-t\right)} N\left(a_{2}\right)\right] } \\
& +\left[S_{t} N_{2}\left(b_{1}, c_{1}, \rho\right)-K_{2} e^{-r\left(T_{2}-t\right)} N_{2}\left(b_{2}, c_{2}, \rho\right)\right] \\
& -\left[S_{t} N_{2}\left(a_{1}, c_{1}, \rho\right)-K_{2} e^{-r\left(T_{2}-t\right)} N_{2}\left(a_{2}, c_{2}, \rho\right)\right] \\
& -A e^{-r\left(T_{1}-t\right)}\left[N\left(b_{2}\right)-N\left(a_{2}\right)\right],
\end{aligned}
$$

where $N(x)$ denotes the standard univariate cumulative normal distribution function, $N_{2}(x, y, \rho)$ denotes the standard bivariate cumulative normal distribution function with a correlation coefficient, $\rho=\sqrt{\left(T_{1}-t\right) /\left(T_{2}-t\right)}$, and

$$
\begin{aligned}
& a_{1}=\frac{\ln \left(S_{t} / H\right)+\left(r+(1 / 2) \sigma^{2}\right)\left(T_{1}-t\right)}{\sigma \sqrt{T_{1}-t}}, \\
& a_{2}=a_{1}-\sigma \sqrt{T_{1}-t}, \\
& b_{1}=\frac{\ln \left(S_{t} / L\right)+\left(r+(1 / 2) \sigma^{2}\right)\left(T_{1}-t\right)}{\sigma \sqrt{T_{1}-t}}, \\
& b_{2}=b_{1}-\sigma \sqrt{T_{1}-t}, \\
& c_{1}=\frac{\ln \left(S_{t} / K_{2}\right)+\left(r+(1 / 2) \sigma^{2}\right)\left(T_{2}-t\right)}{\sigma \sqrt{T_{2}-t}}, \\
& c_{2}=c_{1}-\sigma \sqrt{T_{2}-t} .
\end{aligned}
$$

Longstaff [1] also discusses several special cases of an extendible option, such that if $L>0$ and $H=\infty$, then the extendible option reduces to a compound option on $C\left(S_{t}, K_{2}, T_{2}-T_{1}\right)$ with strike price $A$, and if $L \rightarrow K_{1}$, the value of the extension privilege approaches zero; hence the value of the extendible options is simply $C\left(S_{t}, K_{1}, T_{1}\right)$ (for more discussion on the special cases of the extendible options, see Longstaff [1]).

2.1. Fast Fourier Transform. The FFT approach evaluates an (output) array of the following form:

$$
\begin{aligned}
& Y\left[l_{1}, \ldots, l_{n}\right] \\
& \quad:=\sum_{j_{1}=0}^{N-1} \cdots \sum_{j_{n}=0}^{N-1} e^{-i(2 \pi / N) j_{1} l_{1}-\cdots-i(2 \pi / N) j_{n} l_{n}} X\left[j_{1}, \ldots, j_{n}\right],
\end{aligned}
$$

for any complex (input) array $\left\{X\left[j_{1}, \ldots, j_{n}\right] \in \mathbf{C} \mid j_{1}, \ldots, j_{n}=\right.$ $0, \ldots, N-1\}$. In addition, to implement the FFT method to option pricing, a closed-form representation of the characteristic function is required. For the case of extendible options, we require univariate and bivariate characteristic functions, which are provided by the following lemmas.

Lemma 2. Under the risk-neutral measure $\mathbf{Q}$, the univariate characteristic function is given by

$$
\begin{aligned}
& \varphi_{x_{T_{1}}}\left(u_{1}\right) \\
& =\exp \left\{i u_{1}\left[x_{t}+\left(r-\frac{1}{2} \sigma^{2}\right)\left(T_{1}-t\right)\right]-\frac{1}{2} \sigma^{2}\left(T_{1}-t\right) u_{1}^{2}\right\}, \\
& \varphi_{x_{T_{2}}}\left(u_{2}\right) \\
& =\exp \left\{i u_{2}\left[x_{T_{1}}+\left(r-\frac{1}{2} \sigma^{2}\right)\left(T_{2}-T_{1}\right)\right]\right. \\
& \left.\quad-\frac{1}{2} \sigma^{2}\left(T_{2}-T_{1}\right) u_{2}^{2}\right\} .
\end{aligned}
$$


Lemma 3. Under the risk-neutral measure $\mathbf{Q}$, the bivariate characteristic function is given by

$$
\begin{aligned}
& \varphi_{x_{T_{1}}, x_{T_{2}}}\left(u_{1}, u_{2}\right) \\
& =\exp \left\{i u_{1}\left[x_{t}+\left(r-\frac{1}{2} \sigma^{2}\right)\left(T_{1}-t\right)\right]-\frac{1}{2} u_{1}^{2} \sigma^{2}\left(T_{1}-t\right)\right\} \\
& \quad \times \exp \left\{i u_{2}\left[x_{t}+\left(r-\frac{1}{2} \sigma^{2}\right)\left(T_{2}-t\right)\right]-\frac{1}{2} u_{2}^{2} \sigma^{2}\left(T_{2}-t\right)\right\} .
\end{aligned}
$$

The univariate characteristic function is used in Carr and Madan [5] to price a European option using FFT within the Black-Scholes dynamics. By employing the Radon-Nikodym derivatives, the characteristic function under measure $\mathbf{Q}$ and the spot price measure $\mathbf{Q}_{S}$ are related as follows:

$$
\begin{aligned}
\varphi_{x_{T_{1}}}^{S}\left(u_{1}\right) & =e^{-r\left(T_{1}-t\right)-x_{t}} \mathbf{E}^{\mathbf{Q}}\left[e^{i\left(u_{1}-i\right) x_{T_{1}}} \mid \mathscr{F}_{t}\right], \\
\varphi_{x_{T_{1}}, x_{T_{2}}}^{S}\left(u_{1}, u_{2}\right) & =e^{-r\left(T_{2}-t\right)-x_{t}} \mathbf{E}^{\mathbf{Q}}\left[e^{i u_{1} x_{T_{1}}+i\left(u_{2}-i\right) x_{T_{2}}} \mid \mathscr{F}_{t}\right] .
\end{aligned}
$$

Hence, from (10), we consider the following expectations which can be expressed in integral form as follows:

$$
\begin{aligned}
& \mathbf{E}^{\mathbf{Q}}\left[\mathbf{1}_{\left\{x_{T_{1}}>h\right\}}\right]=\int_{h}^{\infty} p\left(x_{T_{1}}\right) d x_{T_{1}}, \\
& \mathbf{E}^{\mathbf{Q}}\left[\mathbf{1}_{\left\{x_{T_{1}} \geq l, x_{T_{2}} \geq k_{2}\right\}}\right]=\int_{l}^{\infty} \int_{k_{2}}^{\infty} p\left(x_{T_{1}}, x_{T_{2}}\right) d x_{T_{2}} d x_{T_{1}}, \\
& \mathbf{E}^{\mathbf{Q}}\left[\mathbf{1}_{\left\{x_{T_{1}} \geq h, x_{T_{2}} \geq k_{2}\right\}}\right]=\int_{h}^{\infty} \int_{k_{2}}^{\infty} p\left(x_{T_{1}}, x_{T_{2}}\right) d x_{T_{2}} d x_{T_{1}}, \\
& \mathbf{E}^{\mathbf{Q}}\left[\mathbf{1}_{\left\{x_{T_{1}} \geq l\right\}}\right]=\int_{l}^{\infty} p\left(x_{T_{1}}\right) d x_{T_{1}}, \\
& \mathbf{E}^{\mathbf{Q}}\left[\mathbf{1}_{\left\{x_{T_{1}} \geq h\right\}}\right]=\int_{h}^{\infty} p\left(x_{T_{1}}\right) d x_{T_{1}},
\end{aligned}
$$

where $p(\cdot)$ is the probability density of $X$ under the riskneutral probability $\mathbf{Q}$. It is clear that integrals (17), (20), and (21) are similar, so are integrals (18) and (19). Hence we first apply the FFT to integral (18). Following Carr and Madan [5], we multiply (18) by dampening factors $\alpha_{1}$ and $\alpha_{2}$ to ensure square-integrability as follows:

$$
e^{\alpha_{1} l+\alpha_{2} k_{2}} \int_{l}^{\infty} \int_{k_{2}}^{\infty} p\left(x_{T_{1}}, x_{T_{2}}\right) d x_{T_{2}} d x_{T_{1}}, \quad \text { for } \alpha_{1}, \alpha_{2}>0 .
$$

Then, by applying the Fourier transform, we have the following:

$$
\begin{aligned}
& \psi\left(u_{1}, u_{2}\right) \\
& =\iint_{-\infty}^{\infty} e^{i u_{1} l+i u_{2} k_{2}}\left(e^{\alpha_{1} l+\alpha_{2} k_{2}}\right. \\
& \left.\quad \times \int_{l}^{\infty} \int_{k_{2}}^{\infty} p\left(x_{T_{1}}, x_{T_{2}}\right) d x_{T_{2}} d x_{T_{1}}\right) .
\end{aligned}
$$

This can be written in closed-form in terms of the characteristic function as follows:

$$
\psi\left(u_{1}, u_{2}\right)=\frac{\varphi_{x_{T_{1}}, x_{T_{2}}}\left[u_{1}-i \alpha_{1}, u_{2}-i \alpha_{2}\right]}{\left(i u_{1}+\alpha_{1}\right)\left(i u_{2}+\alpha_{2}\right)} .
$$

Using the inverse Fourier transform, we can recover integral (18):

$$
\frac{e^{-\alpha_{1} l-\alpha_{2} k_{2}}}{(2 \pi)^{2}} \iint_{-\infty}^{\infty} e^{-i u_{1} l-i u_{2} k_{2}} \psi\left(u_{1}, u_{2}\right) d u_{2} d u_{1} .
$$

Then, we numerically compute this by invoking the trapezoid rule (see [11]) which approximates the integral by the following sum:

$$
\frac{e^{-\alpha_{1} l-\alpha_{2} k_{2}}}{(2 \pi)^{2}} \sum_{j=0}^{N-1 N-1} \sum_{m=0}^{-i u_{1, j} l-i u_{2, m} k_{2}} \psi\left(u_{1, j}, u_{2, m}\right) \Delta_{2} \Delta_{1},
$$

where $\Delta_{1,2}$ is the distance between the points of the integration grid, $u_{1, j}=(j-(N / 2)) \Delta_{1}$ and $u_{2, m}=(m-$ $(N / 2)) \Delta_{2}$, for $j, m=0, \ldots, N-1$. In order to progress, we define a grid of size $(N \times N)$ by $L_{2}=\left\{\left(l_{u}, k_{2, p}\right) \mid 0 \leq\right.$ $u, p \leq N-1\}$ where $\omega_{1,2}>0$ is the distance between the logarithmic critical prices and the logarithmic strikes, respectively, as follows:

$$
\begin{gathered}
l_{u}=\left(u-\frac{N}{2}\right) \omega_{1}, \\
k_{2, p}=\left(p-\frac{N}{2}\right) \omega_{2} .
\end{gathered}
$$

Hence, we can now evaluate the following sum: $\Gamma\left(l, k_{2}\right)=$ $\sum_{j=0}^{N-1} \sum_{m=0}^{N-1} e^{-i u_{1, j} l-i u_{2, m} k_{2}} \psi\left(u_{1, j}, u_{2, m}\right)$.

By choosing $\omega_{1,2} \Delta_{1,2}=2 \pi / N$ this yields the following values of $\operatorname{sum} \Gamma\left(l, k_{2}\right)$ on $L_{2}$ :

$$
\begin{gathered}
\Gamma\left(l_{u}, k_{2, p}\right) \\
=\sum_{j=0}^{N-1} \sum_{m=0}^{N-1} e^{-i u_{1, j} l_{u}-i u_{2, m} k_{2, p}} \psi\left(u_{1, j}, u_{2, m}\right) \\
=(-1)^{u+p} \sum_{j=0}^{N-1} \sum_{m=0}^{N-1} e^{-i(2 \pi / N) j u-i(2 \pi / N) m p} \\
\times\left[(-1)^{j+m} \psi\left(u_{1, j}, u_{2, m}\right)\right] .
\end{gathered}
$$

We take the (input) array as $X[j, m]=(-1)^{j+m} \psi\left(u_{1, j}\right.$, $\left.u_{2, m}\right)$, for $j, m=0, \ldots, N-1$. Hence, the approximation is at $(N \times N)$ different logarithmic critical prices and strike prices such that

$$
\frac{e^{-\alpha_{1} l_{u}-\alpha_{2} k_{2, p}}}{(2 \pi)^{2}} \Gamma\left(l_{u}, k_{2, p}\right) \Delta_{2} \Delta_{1}, \quad \text { for } 0 \leq u, p \leq N-1 .
$$

In an analogous way, by replacing $l$ with $h$, we can approximate integral (19). The two-dimensional FFT can also be reduced to a one-dimensional FFT which involves integrals (17), (20), and (21). Therefore for brevity, we exclude the development for the one-dimensional FFT in this paper. 
2.2. Monte Carlo Simulation. In this section, we price the extendible call option using Monte Carlo simulation [18]. Given the pricing function of an extendible call option given in (6), the vanilla call option $C$ can be priced at time $T_{1}$ with strike price $K_{2}$ and expiration time $T_{2}$. Thus we only need to compute the asset price until time $T_{1}$. Let $S_{T_{1}, j}$ be the asset price at time $T_{1}$ on the $j$ th path, and let $w_{T_{1}, j}$ be the vanilla call price with strike price $K_{2}$ and expiration time $T_{2}$ at time $T_{1}$ for the $j$ th path; that is, $C\left(S_{T_{1}, j}, K_{2}, T_{2}-T_{1}\right)$. Recall the closed-form solution for a vanilla call option, so we have

$$
C=S_{T_{1}} N\left(e_{1}\right)-K_{2} e^{-r\left(T_{2}-T_{1}\right)} N\left(e_{2}\right),
$$

where

$$
\begin{gathered}
e_{1}=\frac{\ln \left(S_{T_{1}} / K_{2}\right)+\left(r+(1 / 2) \sigma^{2}\right)\left(T_{2}-T_{1}\right)}{\sigma \sqrt{T_{2}-T_{1}}}, \\
e_{2}=e_{1}-\sigma \sqrt{T_{2}-T_{1}} .
\end{gathered}
$$

Therefore, the Monte Carlo estimator for the price of an extendible call option is given by

$$
\operatorname{EC}\left(t, \widehat{x}_{T_{1}}\right)=\frac{e^{-r\left(T_{1}-t\right)}}{n} \sum_{j=1}^{n} \max \left[e^{\hat{x}_{T_{1}}^{j}}-K_{1}, w_{T_{1}, j}-A, 0\right],
$$

where $n$ is the number of simulations. Suppose we have $x_{t}=$ $\ln S_{t}$ with the following process:

$$
x_{t}=\left(r-\frac{1}{2} \sigma^{2}\right) d t+\sigma d W_{t} .
$$

We discretize the asset path using the Euler scheme as follows:

$$
\begin{gathered}
x_{j+1}=x_{j}+\left(r-\frac{1}{2} \sigma^{2}\right) \Delta t+\sigma \Delta W_{j}, \quad t_{j}=j \Delta t, \\
\Delta W_{j}=W_{t_{j+1}}-W_{t_{j}}=Z \sqrt{\Delta t} \quad \text { with } Z \sim N(0,1) .
\end{gathered}
$$

We also apply the Milstein scheme [19] that is strongly convergent with order one such that

$$
\begin{array}{r}
x_{j+1}=x_{j}+\left(r-\frac{1}{2} \sigma^{2}\right) \Delta t+\sigma \Delta W_{j}+\frac{1}{2} \sigma^{2}\left((\Delta W)^{2}-\Delta t\right), \\
t_{j}=j \Delta t \\
\Delta W_{j}=W_{t_{j+1}}-W_{t_{j}}=Z \sqrt{\Delta t} \quad \text { with } Z \sim N(0,1) .
\end{array}
$$

We let $\left[t=t_{0}<t_{1}<\cdots<t_{m}=T\right]$ be a partition of the time interval $[t, T]$ of $m$ equal segments with length $\Delta t_{j}$, where $\Delta t_{j}=t_{j+1}-t_{j}$ and $t_{j}=j T / m$, for each $j=1, \ldots, M$. We draw a random sample from a normal distribution with mean 0 and volatility 1 for each $j=0,1, \ldots, m$ to simulate (34) and (35), thus generating a sample path for $x_{T}$ by simulating $x_{j}$ for $j=1$ to $j=m$. This step is repeated to generate many paths. We then estimate the price of the extendible call option with Monte Carlo simulation.
TABLE 1: Inputs to price the extendible options.

\begin{tabular}{lc}
\hline Input & Value \\
\hline Initial strike price, $K_{1}$ & 100 \\
Initial expiration time, $T_{1}$ & 0.5 \\
Extended strike price, $K_{2}$ & 105 \\
Extended expiration time $T_{2}$ & 0.75 \\
Risk-free rate, $r$ & 0.08 \\
Premium, $A$ & 1 \\
\hline
\end{tabular}

\section{Numerical Results}

In this section, we present a numerical comparison between the FFT and the Monte Carlo simulation techniques which have been described in previous sections. (The computations were implemented in MATLAB and conducted on an Intel Core 2 Quad processor Q8200 @ 2.33 GHz machine running under Windows 7 Professional with 4 GB RAM).

We price the extendible call option for a range of underlying asset prices, $S_{t}=80,90,100,110,120$, and $\sigma=0.25$, with the inputs in Table 1 . We found that the dampening factor $\alpha$ differs for a one-dimensional and a two-dimensional FFT. For the one-dimensional FFT, we choose $\alpha=1.75$ and $\Delta=0.3$, while for the two-dimensional FFT, $\alpha=\{0.75,1.75\}$ and $\Delta=$ 0.2. Table 2 provides the CPU time taken by the FFT, Euler scheme, and Milstein scheme to produce the prices for extendible call options. It can be seen that FFT takes less than half a second, on average, approximately 0.0869 seconds, to compute the $N=2^{7}$ prices in a single computation, while the exact solution computed each of the option prices instantaneously. Euler takes slightly longer than a minute, on average 75.4870 seconds, whereas Milstein scheme takes the longest time to produce results, 311.0502 seconds on average. In Table 3, we document the prices obtained using Monte Carlo simulations with Euler and Milstein schemes. The exact solution is used to compute the relative errors between these two schemes. The error is given by the following:

$$
\epsilon \approx \frac{\left|\mathrm{EC}_{\text {True }}-\mathrm{EC}_{\mathrm{Approx}}\right|}{\mathrm{EC}_{\text {True }}} \times 100,
$$

where $\mathrm{EC}_{\text {True }}$ is the Black-Scholes pricing solution and $\mathrm{EC}_{\text {Approx }}$ is the estimation from the simulation. From the table, it can be seen that the relative error for the Euler scheme is smaller than the Milstein scheme. We also plot the price convergence of the Monte Carlo simulation when the underlying asset $S=90$ in Figure 2. From Tables 2 and 3, it can be seen that the Euler scheme is more computationally efficient than the Milstein scheme. According to Platen and Bruti-Liberati [20], the Euler scheme has fewer terms and the same order of weak convergence; hence, apart from efficiency issue, the Milstein scheme also has numerical stability issue which can be worse than that of the Euler scheme. We then compare the extendible call prices computed by the FFT and the exact solution. Table 4 shows the prices and percentage differences taken relative to the FFT prices. 


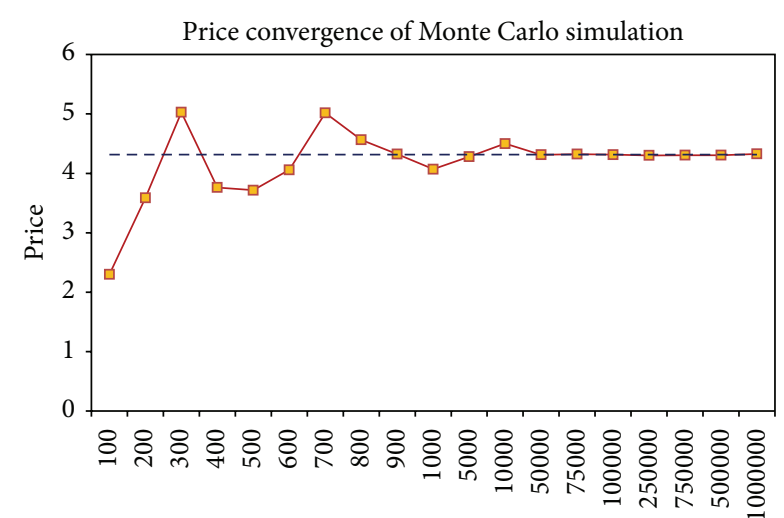

Number of simulations

$\rightarrow-$ Price

- - Analytical price

Figure 2: Price convergence of Monte Carlo simulation for extendible call options for $S=90$.

TABLE 2: CPU time (in seconds) for pricing an extendible call option: FFT, Euler scheme, and Milstein scheme.

\begin{tabular}{lccc}
\hline Underlying asset, $S$ & FFT & Euler scheme & Milstein scheme \\
\hline 80 & 0.0821 & 71.4400 & 289.2060 \\
90 & 0.0927 & 82.0390 & 310.9170 \\
100 & 0.1042 & 81.9460 & 331.771 \\
110 & 0.0912 & 77.6070 & 359.385 \\
120 & 0.0643 & 64.4030 & 263.972 \\
\hline
\end{tabular}

TABLE 3: Prices and relative error (in percent) for pricing an extendible call option: exact solution, Euler scheme, and Milstein scheme.

\begin{tabular}{lccccc}
\hline $\begin{array}{l}\text { Underlying } \\
\text { asset, } S\end{array}$ & $\begin{array}{c}\text { Exact } \\
\text { solution }\end{array}$ & Euler price & $\epsilon$ & $\begin{array}{c}\text { Milstein } \\
\text { Price }\end{array}$ & $\epsilon$ \\
\hline 80 & 1.4264 & 1.4294 & 0.2071 & 1.8025 & 26.3641 \\
90 & 4.3154 & 4.3282 & 0.2982 & 4.6685 & 8.1827 \\
100 & 9.4228 & 9.3505 & 0.7679 & 9.4874 & 0.6851 \\
110 & 16.5358 & 16.5445 & 0.0524 & 16.3863 & 0.9040 \\
120 & 25.0506 & 25.0283 & 0.0888 & 24.8268 & 0.8933 \\
\hline
\end{tabular}

TABle 4: Prices and relative error (in percent) for pricing an extendible call option: exact solution and FFT.

\begin{tabular}{lccc}
\hline Underlying asset, $S$ & Exact solution & FFT price & $\epsilon$ \\
\hline 80 & 1.4264 & 1.4247 & 0.1193 \\
90 & 4.3154 & 4.2908 & 0.5733 \\
100 & 9.4228 & 9.5106 & 0.9232 \\
110 & 16.5358 & 16.4352 & 0.6121 \\
120 & 25.0506 & 25.1067 & 0.2234 \\
\hline
\end{tabular}

\section{Conclusion}

This paper studies the pricing of extendible options under the Black and Scholes [16] model by developing a pricing framework which utilizes the FFT technique. The availability of a closed-form representation of the characteristic function enables us to implement the FFT. The characteristic function for the extendible options involves a univariate and a bivariate characteristic function. Hence, the application of the FFT technique involves a one-dimensional and a two-dimensional FFT algorithm.

Additionally, we computed the prices of the extendible options using Monte Carlo simulation and used the closedform pricing solution as a benchmark to compare the numerical efficiency of the FFT and the Monte Carlo simulation. The FFT produces a range of option prices for a range of strike prices for a given maturity. Moreover, the numerical examples show that the FFT is more computationally efficient than the Monte Carlo simulation.

Dias and Rocha [2] show that the framework of extendible options can be applied to model oil prices using mean reversion with jumps. Hence, further development such as pricing extendible options with other stochastic features such as stochastic interest rate can be applied. In the future, we plan to use the FFT to price extendible options under the Heston stochastic volatility model [21].

The work presented here can also be extended to a more general case, that is, for $n$-extensions. The FFT has become well established as an option pricing tool since it was first introduced by Carr and Madan [5]. Fang and Oosterlee [22] have introduced a Fourier-Cosine also known as the COS method to price options, and this method has been shown to be faster under certain circumstances. Hence, another possible development of this work is to price extendible options using the COS method and compare the performance of the two Fourier methods in the case for extendible options.

\section{Conflict of Interests}

The authors declare that there is no conflict of interests regarding the publication of this paper.

\section{Acknowledgments}

Siti Nur Iqmal Ibrahim's research was supported by Universiti Putra Malaysia and the Ministry of Higher Education, Malaysia. The authors would like to thank the editor and the referee for their constructive comments that led to a significant improvement of the paper.

\section{References}

[1] F. A. Longstaff, "Pricing options with extendible maturities: analysis and applications," The Journal of Finance, vol. 45, no. 3, pp. 935-957, 1990.

[2] M. A. Dias and K. M. C. Rocha, "Petroleum concession with extendible options using mean reversion with jumps to model oil prices," Working Paper, IPEA, Rio de Janeiro, Brazil, 2000.

[3] Y. P. Chung and H. Johnson, "Extendible options: the general case," Finance Research Letters, vol. 8, no. 1, pp. 15-20, 2011.

[4] J. W. Cooley and J. W. Tukey, "An algorithm for the machine calculation of complex Fourier series," Mathematics of Computation, vol. 19, no. 90, pp. 297-301, 1965. 
[5] P. Carr and D. Madan, "Option valuation using the fast Fourier transform," Journal of Computational Finance, vol. 2, no. 4, pp. 61-73, 1999.

[6] E. Pillay and J. G. O'Hara, "FFT based option pricing under a mean reverting process with stochastic volatility and jumps," Journal of Computational and Applied Mathematics, vol. 235, no. 12, pp. 3378-3384, 2011.

[7] S. N. I. Ibrahim, J. G. O'Hara, and N. Constantinou, "Power option pricing via fast Fourier transform," in Proceedings of the 4th Computer Science and Electronic Engineering Conference (CEEC '12), pp. 1-6, Colchester, UK, September 2012.

[8] E. Eberlein, K. Glau, and A. Papapantoleon, "Analysis of Fourier transform valuation formulas and applications," Applied Mathematical Finance, vol. 17, no. 3, pp. 211-240, 2010.

[9] U. Cherubini, G. D. Lunga, S. Mulinacci, and P. Rossi, Fourier Transform Methods in Finance, John Wiley \& Sons, 2010.

[10] E. Benhamou, "Fast Fourier transform for discrete Asian options," Journal of Computational Finance, vol. 6, pp. 49-61, 2002.

[11] M. A. H. Dempster and S. S. G. Hong, "Spread option valuation and the fast Fourier transform," in Mathematical FinanceBachelier Congress 2000, pp. 203-220, Springer, Berlin, Germany, 2002.

[12] A. Esser, "General valuation principles for arbitrary payoffs and applications to power options under stochastic volatility," Financial Markets and Portfolio Management, vol. 17, no. 3, pp. 351-372, 2003.

[13] S.-M. Zhang and L.-H. Wang, "A fast Fourier transform technique for pricing European options with stochastic volatility and jump risk," Mathematical Problems in Engineering, vol. 2012, Article ID 761637, 17 pages, 2012.

[14] S. N. I. Ibrahim, J. G. O’Hara, and N. Constantinou, “Pricing power options under the Heston dynamics using the FFT," New Trends in Mathematical Sciences, vol. 1, no. 1, pp. 1-9, 2013.

[15] J. Huang, W. Zhu, and X. Ruan, "Fast Fourier transform based power option pricing with stochastic interest rate, volatility, and jump intensity," Journal of Applied Mathematics, vol. 2013, Article ID 875606, 7 pages, 2013.

[16] F. Black and M. Scholes, "The pricing of options and corporate liabilities," Journal of Political Economy, vol. 81, no. 3, pp. 637659, 1973.

[17] C. R. Gukhal, "The compound option approach to American options on jump-diffusions," Journal of Economic Dynamics and Control, vol. 28, no. 10, pp. 2055-2074, 2004.

[18] P. P. Boyle, "Options: a Monte Carlo approach," Journal of Financial Economics, vol. 4, no. 3, pp. 323-338, 1977.

[19] G. N. Mil'shtein, "Approximate integration of stochastic differential equations," Theory of Probability and Its Applications, vol. 19, no. 3, pp. 557-562, 1974.

[20] E. Platen and N. Bruti-Liberati, Numerical Solution of Stochastic Differential Equations with Jumps in Finance, vol. 64 of Stochastic Modelling and Applied Probability, Springer, Berlin, Germany, 2010.

[21] S. L. Heston, "A closed-form solution for options with stochastic volatility with applications to bond and currency options," Review of Financial Studies, vol. 6, no. 2, pp. 327-343, 1993.

[22] F. Fang and C. W. Oosterlee, "A Fourier-based valuation method for bermudan and barrier options under Heston's model," SIAM Journal on Financial Mathematics, vol. 2, no. 1, pp. 439-463, 2011. 


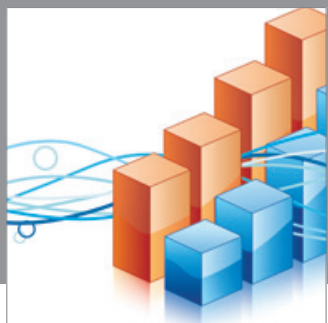

Advances in

Operations Research

mansans

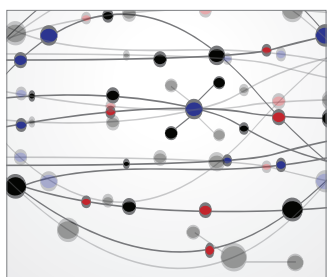

The Scientific World Journal
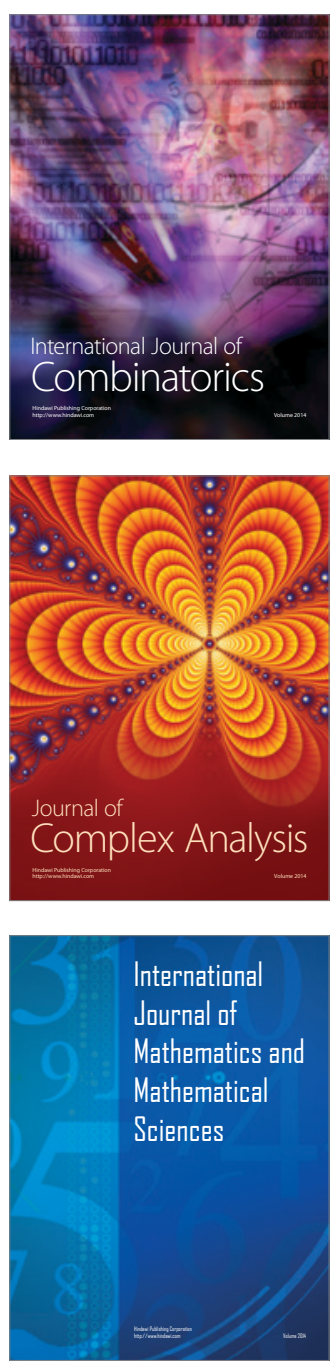
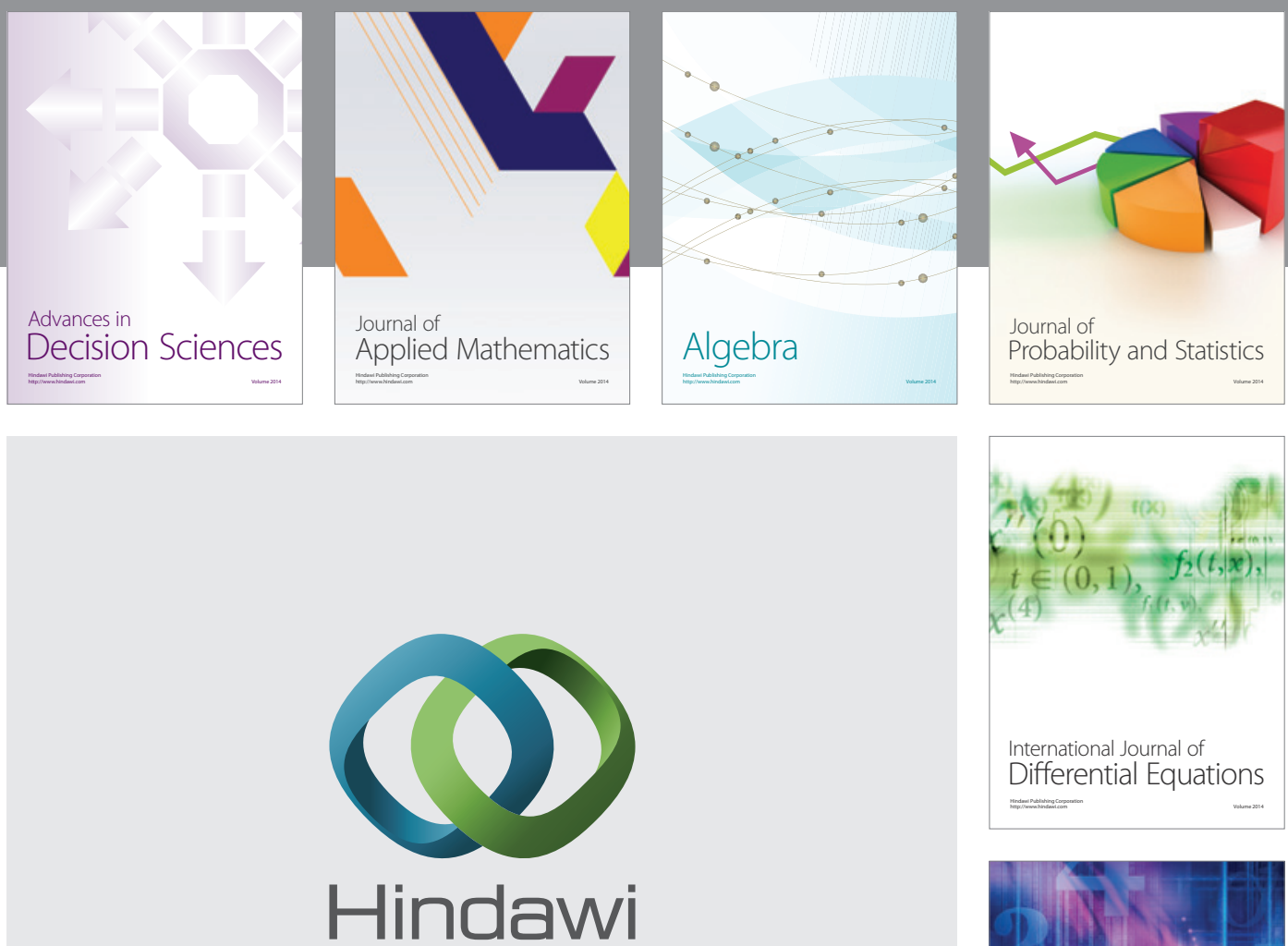

Submit your manuscripts at http://www.hindawi.com
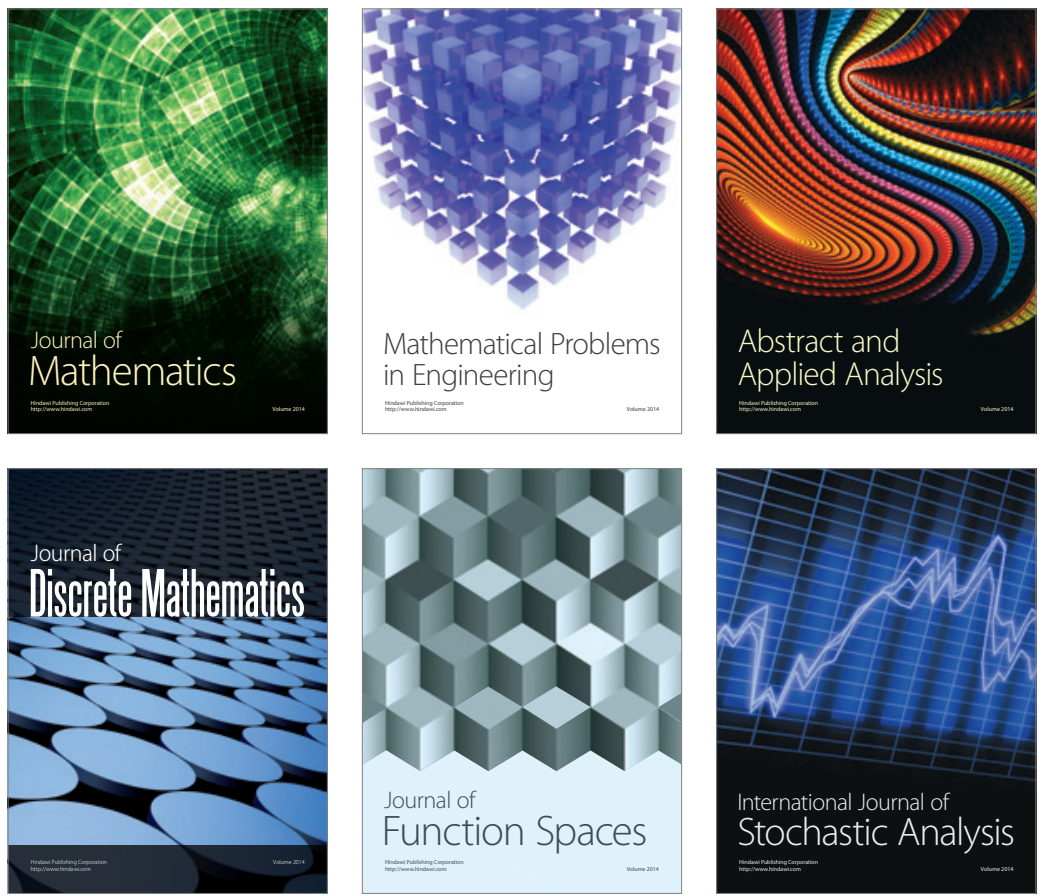

Journal of

Function Spaces

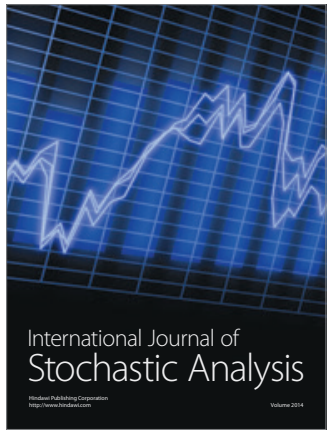

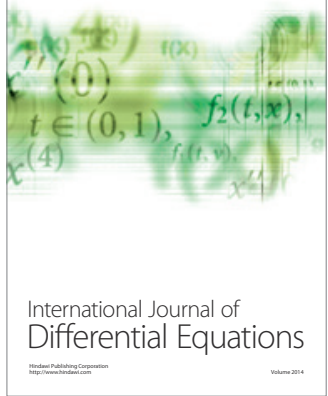
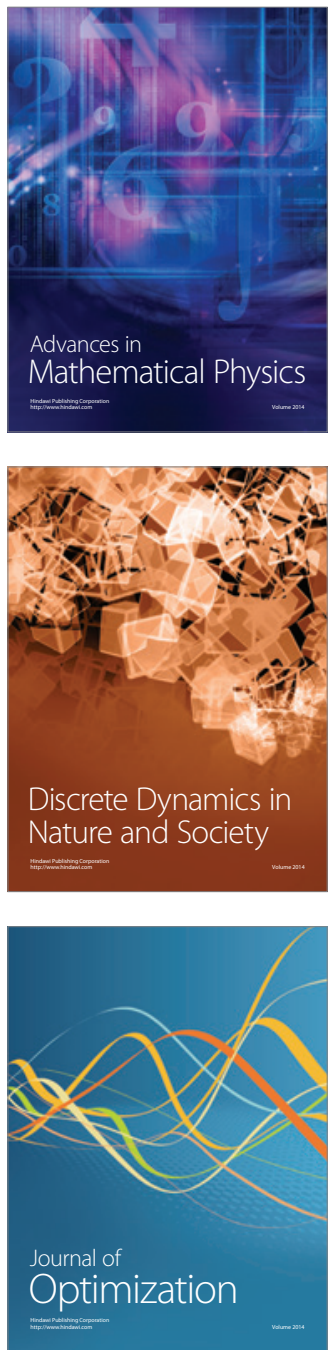